Original Research Paper

\title{
Exponentiated Exponential Weibull Distribution: Mathematical Properties and Application
}

\author{
Dawlah Al-Sulami \\ Department of Statistics, Faculty of Science, King Abdulaziz University, Jeddah 21589, Saudi Arabia
}

\author{
Article history \\ Received: $30-05-2020$ \\ Revised: 31-08-2020 \\ Accepted: 03-09-2020
}

Email: dalsulami@kau.edu.sa

\begin{abstract}
An Exponentiated Exponential Weibull Distribution (EEWD) is proposed. Some statistical properties of the proposed distribution are studied. The Maximum Likelihood method (ML) is applied to estimate the model parameters. The efficiency of the ML estimates of the proposed model is demonstrated using a Monte Carlo simulation study with different samples sizes. Moreover, real data set application is provided to show the flexibility of the proposed model in comparison to some selected distributions.
\end{abstract}

Keywords: Transformed-Transformer Family, Exponential-G Family, Exponentiated Distribution, Weibull Distribution, Maximum Likelihood Estimation

\section{Introduction}

Statistical distributions are of vital importance in fitting data in real phenomena. It is widely applied to model and analyze data in different disciplines such as engineering, biology, economics, finance and medical sciences. They, occasionally, work well with different types of data; however, in many instances, they are not flexible enough to analyze the complex behaviour displayed by data. Therefore, the attention has, recently, been drawn to suggest more flexible distributions, which have the ability to fit any kind of data with any degree of complexity. Adding an extra parameter to any existing distribution is one way to obtain more flexible and adaptable distribution. This method proposed by (Gupta et al., 1998) and named the exponentiated method as the Cumulative Distribution Function (cdf) of any distribution has been raised to a parameter; hence, a new flexible distribution is generated. Let $X$ be a random variable for any continuous distribution with cdf $G(x)$, thus the cdf of the exponentiated distribution is given by:

$$
F(x)=[G(x)]^{\alpha}
$$

where, $\alpha$ is an extra shape (power) parameter. Many researchers have started to apply the exponentiated method on different traditional distributions. For example, (Gupta and Kundu, 2001) presented the exponentiated exponential (also called generalized exponential) distribution, (Nadarajah and Kotz, 2006) introduced some new exponentiated distributions, such as Gumbel, Weibull and Gamma distributions, (El-Gohary et al., 2013) proposed the generalized Gompertz distribution, (Sarhan and Apaloo, 2013) suggested the exponentiated modified Weibull extension distribution, (Abu-Zinadah and Aloufi, 2014) introduced the exponentiated Gompertz distribution and (Rather and Subramanian, 2018) considered the exponentiated Mukhrejee-Islam distribution; among others.

Generating new families of distributions is another powerful technique to develop more flexible distributions. For any continuous distribution, different types of generators can be applied to obtain new families of distributions. Alzaatreh et al. (2013) proposed transformed-transformer $\mathrm{T}-\mathrm{X}$ family which has become increasingly used due to its flexibility as any continuous distribution can be chosen as a generator. Let $X$ be a random variable for any continuous distribution, then the cdf for the T-X family is given by:

$$
F(x, \zeta)=\int_{a}^{W(G(x, \zeta))} r(t) d t
$$

where, $G(x, \zeta)$ is the cdf of any baseline distribution with vector parameters $\zeta, W(\cdot)$ is a function of the cdf $G(x, \zeta)$ and $r(t)$ is the pdf of a random variable $T$. By using different forms of the transformer $W(G(x, \zeta))$, new class of T-X family of distributions will be obtained. Recently, Some attempts have been made to generate new distributions with different form of $W(G(x, \zeta))$. For example, (Bourguignon et al., 2014) proposed the 
Weibull-G family of probability distributions, (Torabi and Montazeri, 2014) introduced the logistic-G family, (Tahir et al., 2015) constructed the logistic-G family, (Hassan and Elgarhy, 2016) obtained the Kumaraswamy Weibull-G family, (Klakattawi, 2019) proposed Weibullgamma distribution and (Afify et al., 2020) generated the Weibull Marshall-Olkin Lindley distribution.

Weibull distribution is considered the most popular statistical distributions for modelling lifetime data. If $X$ is a random variable from Weibull distribution with parameters $c, \beta>0$, then the pdf and cdf are respectively given by:

$$
\begin{aligned}
& g(x ; c, \beta)=\frac{c}{\beta}\left(\frac{x}{\beta}\right)^{c-1} e^{-\left(\frac{x}{\beta}\right)^{c}} ; x \geq 0 \\
& G(x ; c, \beta)=1-e^{-}\left(\frac{x}{\beta}\right)^{c}
\end{aligned}
$$

Weibull distribution is widely preferable in reliability studies although its monotonic hazard function is not suitable for many lifetime data. Hence, a modification of Weibull distribution has been suggested by many researchers. For instance, (Mudholkar and Srivastava, 1993) proposed the Exponentiated Weibull family, (Lai et al., 2003) and (Sarhan and Zaindin, 2009) introduced modified Weibull distribution, (Lee et al., 2007) studied Beta-Weibull distribution, (Bebbington et al., 2007) suggested a flexible Weibull distribution, (Carrasco et al., 2008) proposed generalized modified Weibull distribution, (Silva et al., 2010) and (Nadarajah et al., 2011) suggested Beta modified Weibull distribution and (Singla et al., 2012) studied the mathematical properties of the Beta generalized Weibull distribution.

In this study, we propose an Exponentiated Exponential Weibull Distribution (EEWD) based on the idea of (Alzaatreh et al., 2013) with $W(G(x, \zeta))=$ $-\log [1-G(x, \zeta)]$. The rest of this paper is organized as follows. Section (2) discusses the EEWD with some special cases. The statistical properties of the proposed model are given in section (3). The distribution of the order statistics of the EEWD discussed in section (4). The maximum likelihood estimates of the EEWD's parameters are given in section (5). The performance of the proposed model is studied in section (6) using a Monte Carlo simulation study and a real data to illustrates the flexibility of the new distribution compared to some distributions in literature. Finally, section (7) concludes.

\section{Construction of the Exponentiated Exponential Distribution}

In this section, we introduce the EEWD. If $t$ is a random variable from the exponential distribution, then cdf of the exponential Weibull distribution is given by:

$H(x, \zeta)=\int_{0}^{-\log [1-G(x, \zeta)]} \lambda e^{-\lambda t} d t$

where $G(x, \zeta)$ is the cdf of the Weibull distribution given by Equation (3). To complete constructing the EEWD, the cdf in (4) will be raised to a parameter $\alpha$. Thus, the EEWD has a cdf given by:

$F(x ; c, \beta, \alpha, \lambda)=\left[1-e^{-\lambda\left(\frac{x}{\beta}\right)^{c}}\right]^{\alpha} ; x>0, c>0, \beta>0, \alpha>0, \lambda>0$

and the corresponding pdf takes the form:

$$
\begin{aligned}
& f(x ; c, \beta, \alpha, \lambda)=\frac{\alpha \lambda c}{\beta}\left(\frac{x}{\beta}\right)^{c-1} e^{-\lambda\left(\frac{x}{\beta}\right)^{c}}\left[1-e^{-\lambda\left(\frac{x}{\beta}\right)^{c}}\right]^{\alpha-1} \\
& ; \quad x>0, c>0, \beta>0, \alpha>0, \lambda>0
\end{aligned}
$$

The survival function and hazard rate function of the random variable $X$ are respectively given by:

$$
\begin{aligned}
& S(x ; c, \beta, \alpha, \lambda)=1-\left[1-e^{\left.-\lambda\left(\frac{x}{\beta}\right)^{c}\right]^{\alpha}}\right. \\
& h(x ; c, \beta, \alpha, \lambda)=\frac{\frac{\alpha \lambda c}{\beta}\left(\frac{x}{\beta}\right)^{c-1} e^{-\lambda}\left(\frac{x}{\beta}\right)^{c}\left[1-e^{-\lambda}\left(\frac{x}{\beta}\right)^{c}\right]^{\alpha-1}}{1-\left[1-e^{-\lambda\left(\frac{x}{\beta}\right)^{c}}\right]^{\alpha}}
\end{aligned}
$$

The pdf, cdf, survival and hazard function for different parameters values are provided in Fig. 1.

\section{Special Cases of the EEWD}

The four parameters EEWD include some known distributions as special cases. Here we introduced some special cases from the proposed EEWD. In Equation (6):

1. If $\alpha=\lambda=1$, the Weibull distribution is obtained

2. If $c=\beta=1$, the exponentiated exponential Weibull distribution is reduced to the generalized exponential distribution

3. If $\alpha=c=\beta=1$, we get the exponential distribution

4. If $\lambda=1$, the generalized Weibull distribution yields

5. If $c=2, \lambda=1$, we obtain Burr type $X$ distribution 

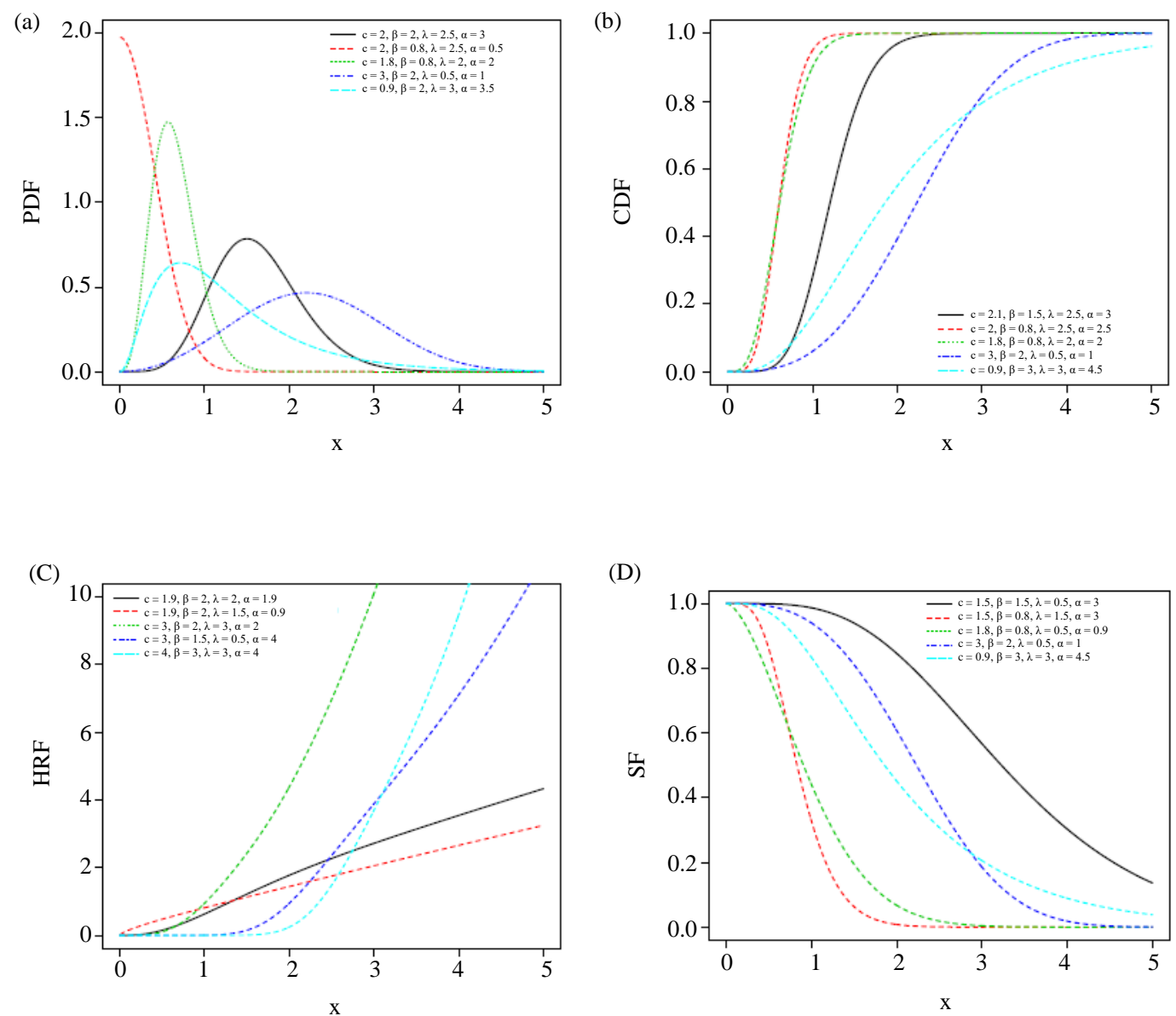

Fig. 1: Plot of: (a) pdf, (b) cdf, (c) hazard function and (d) survival function with different values of $c, \beta, \lambda, \alpha$

\section{Statistical Properties}

This section provides some mathematical properties of the proposed EEWD. Studying the mathematical properties gives a clear insight about the distribution. In particular, the quantile, median, moments, incomplete moments and moments generating function are obtained in this section.

\section{Quantile and Median}

For the random variable $\mathrm{X}$ from the EEWD, the quantile $F\left(x_{q}\right)=q ; 0<q<1$ can be written in an explicit form and is given by $F\left(x_{q}\right)=q$, thus:

$$
x_{q}=\beta\left[\log \frac{1}{\left(1-q^{\frac{1}{\alpha}}\right)^{\frac{1}{\lambda}}}\right]^{\frac{1}{c}}
$$

and, hence, the median is obtained by taking $q=0.5$, therefore:

$$
x_{(0.5)}=\beta\left[\log \frac{1}{\left(1-(0.5)^{\frac{1}{\alpha}}\right)^{\frac{1}{\lambda}}}\right]^{\frac{1}{c}}
$$

\section{Moments and Generating Function}

Moments are of great importance to understand the most important characteristics of the statistical distribution. They are helpful to get a deep idea about the mean, variance, skewness and kurtosis of the lifetime data. In this subsection, the moments, including mean and variance, of the EEWD as well as the incomplete moment are obtained. Moreover, the moment generating function is introduced.

\section{Moments}

If $X$ is a random variable from the EEWD, the $r$ th moments is given by: 


$$
\begin{aligned}
& \mu_{r}^{\prime}=E\left(X^{r}\right)=\int_{0}^{\infty} x^{r} \frac{\alpha \lambda c}{\beta}\left(\frac{x}{\beta}\right)^{c-1} e^{-\lambda\left(\frac{x}{\beta}\right)^{c}}\left[1-e^{-\lambda\left(\frac{x}{\beta}\right)^{c}}\right]^{\alpha-1} d x \\
& =\sum_{i=0}^{\infty}(-1)^{i}\left(\begin{array}{c}
\alpha-1 \\
i
\end{array}\right) \frac{\alpha \beta^{r}}{\lambda^{\frac{r}{c}}(i+1)^{\frac{r}{c}+1}} \Gamma\left(\frac{r}{c}+1\right)
\end{aligned}
$$

Now let:

$\omega_{r}(c)=\sum_{i=0}^{\infty}(-1)^{i}\left(\begin{array}{c}\alpha-1 \\ i\end{array}\right) \frac{1}{(i+1)^{\frac{r}{c}+1}}$

then the $r$ th moment of the EEWD is given by:

$$
\mu_{r}^{\prime}=a \lambda^{-\frac{r}{c}} \omega_{r}(c) \beta^{r} \Gamma\left(\frac{r}{c}+1\right)=a \lambda^{-\frac{r}{c}} \omega_{r}(c) E^{W}\left(x^{r}\right)
$$

where, $E^{W}\left(x_{r}\right)$ is the $r$ th moment of the Weibull distribution. Therefore, the mean of the EEWD takes the form:

$$
\begin{aligned}
& E(X)=\sum_{i=0}^{\infty}(-1)^{i}\left(\begin{array}{c}
\alpha-1 \\
i
\end{array}\right) \frac{\alpha \beta}{\lambda^{\frac{1}{c}}(i+1)^{\frac{1}{c}+1}} \Gamma\left(\frac{1}{c}+1\right) \\
& =\alpha \lambda^{-\frac{1}{c}} \omega_{1}(c) E^{W}(x)
\end{aligned}
$$

where, $E^{W}(x)$ is the mean of Weibull distribution. The variance of the EEWD is calculated from the following relation:

$$
\operatorname{Var}(X)=E\left(X^{2}\right)-[E(X)]^{2}
$$

where, $E\left(x^{2}\right)$ is the second moment obtained by putting $r$ $=2$ in (8), thus:

$$
\begin{aligned}
& E\left(X^{2}\right)=\sum_{i=0}^{\infty}(-1)^{i}\left(\begin{array}{c}
\alpha-1 \\
i
\end{array}\right) \frac{\alpha \beta^{2}}{\lambda^{\frac{2}{c}}(i+1)^{\frac{2}{c}+1}} \Gamma\left(\frac{2}{c}+1\right) \\
& =\alpha \lambda^{-\frac{2}{c}} \omega_{2}(c) E^{W}\left(x^{2}\right)
\end{aligned}
$$

Thus, from (9) and (10), the variance is expressed as:

$$
\operatorname{Var}(X)=\alpha \lambda^{-\frac{2}{c}} \omega_{2}(c) E^{W}\left(x^{2}\right)-\left[\alpha \lambda^{-\frac{1}{c}} \omega_{1}(c) E^{W}(x)\right]^{2}
$$

\section{Incomplete Moments}

The $r$ th incomplete moments of the EEWD is written as:

$$
\begin{aligned}
& \varphi_{X}(t)=\int_{a}^{\infty} x^{r} \frac{\alpha \lambda c}{\beta}\left(\frac{x}{\beta}\right)^{c-1} e^{-\lambda\left(\frac{x}{\beta}\right)^{c}}\left[1-e^{-\lambda\left(\frac{x}{\beta}\right)^{c}}\right]^{\alpha-1} d x \\
& =\sum_{i=0}^{\infty}(-1)^{i}\left(\begin{array}{c}
\alpha-1 \\
i
\end{array}\right) \frac{\alpha \beta^{r}}{\lambda^{\frac{r}{c}}(i+1)^{\frac{r}{c}+1}} \Gamma\left(\frac{r}{c}+1, \lambda\left(\frac{a}{\beta}\right)^{c}\right) \\
& =\alpha \lambda^{-\frac{r}{c}} \omega_{r}(c) \beta^{r} \Gamma\left(\frac{r}{c}+1, \lambda\left(\frac{a}{\beta}\right)^{c}\right)
\end{aligned}
$$

\section{Moment Generating Function}

The moment generating function of the EEWD can be obtained as:

$M_{x}(t)=\int_{0}^{\infty} e^{t x} \frac{\alpha \lambda c}{\beta}\left(\frac{x}{\beta}\right)^{c-1} e^{-\lambda\left(\frac{x}{\beta}\right)^{c}}\left[1-e^{-\lambda\left(\frac{x}{\beta}\right)^{c}}\right]^{\alpha-1} d x=\sum_{r=0}^{\infty} \frac{t^{r}}{r} \mu_{r}^{\prime}$

$=\sum_{r=0}^{\infty} \frac{t^{r} \alpha \lambda^{-\frac{r}{c}}}{r !} \omega_{r}(c) E^{W}\left(x^{r}\right)$

\section{Order Statistics}

Let $x_{1: n}, x_{2: \mathrm{n}}, \ldots, x_{n: n}$ be the order statistics obtained from a random sample $x_{1}, x_{2}, \ldots, x_{n}$ from the EEWD with pdf $f(x)$ and cdf $F(x)$, respectively, given by (6) and (5). Then the pdf of the $r$ th order statistics can be expressed as:

$$
\begin{aligned}
& f_{r: n}(x)=\frac{n !}{(r-1) !(n-r) !}[F(x)]^{r-1} \\
& {[1-F(x)]^{n-r} f(x) ; r=1,2, \ldots, n}
\end{aligned}
$$

by using binomail expansion:

$$
[1-F(x)]^{n-r}=\sum_{i=0}^{n-r}(-1)^{i}\left(\begin{array}{c}
n-r \\
i
\end{array}\right)[F(x)]^{i}
$$

Thus, by substituting (6) and (5) into (14) and using the binomial expansion, the pdf of the $r$ th order statistics of the EEWD is written as:

$$
\begin{aligned}
& f_{r: n}(x)=\sum_{i=0}^{n-r} \frac{(-1)^{i} n !}{i !(r-1) !(n-r-i) !} \frac{\alpha \lambda c}{\beta}\left(\frac{x}{\beta}\right)^{c-1} \\
& e^{-\lambda\left(\frac{x}{\beta}\right)}\left[1-e^{-\lambda\left(\frac{x}{\beta}\right)}\right]^{\alpha(r+i)-1}
\end{aligned}
$$

Therefore, the pdf of the smallest order statistic $x_{(1)}$ is given by: 


$$
\begin{aligned}
& f_{1: n}(x)=\sum_{i=0}^{n-1} \frac{(-1)^{i} n !}{i !(n-1-i) !} \frac{\alpha \lambda c}{\beta}\left(\frac{x}{\beta}\right)^{c-1} \\
& e^{-\lambda\left(\frac{x}{\beta}\right)^{c}}\left[1-e^{-\lambda\left(\frac{x}{\beta}\right)^{c}}\right]^{\alpha(1+i)-1}
\end{aligned}
$$

Moreover, the pdf of the largest order statistic $x_{(n)}$ is given by:

$$
f_{n: n}(x)=n \frac{\alpha \lambda c}{\beta}\left(\frac{x}{\beta}\right)^{c-1} e^{-\lambda\left(\frac{x}{\beta}\right)^{c}}\left[1-e^{-\lambda\left(\frac{x}{\beta}\right)^{c}}\right]^{\alpha n-1}
$$

\section{Parameters Estimation}

In this section, the Maximum Likelihood (ML) method will be applied to estimate the parameters $c, \beta, \lambda$ and $\alpha$ of the EEWD. For a random sample $x_{1}, x_{2}, \ldots, x_{n}$ from a EEWD, the likelihood function is given by:

$$
L=\prod_{i=1}^{n} f\left(x_{i} ; c, \beta, \lambda, \alpha\right)
$$

Now by substituting from (6) into (18) and taking the $\log$, the $\log$ likelihood function is expressed as:

$$
\begin{aligned}
& \ell=n \log (a)+n \log (\lambda)+n \log (c) \\
& -n \log (\beta)+(c-1) \sum_{i=1}^{n} \log \left(\frac{x_{i}}{\beta}\right) \\
& -\lambda \sum_{i=1}^{n}\left(\frac{x_{i}}{\beta}\right)^{c}+(\alpha-1) \sum_{i=1}^{n} \log \left[1-e^{-\lambda\left(\frac{x_{i}}{\beta}\right)^{c}}\right]
\end{aligned}
$$

The derivative of Equation (19) with respect to parameters $c, \beta, \lambda, \alpha$ is; respectively, given by:

$$
\begin{aligned}
& \frac{\partial \ell}{\partial c}=\frac{n}{c}+\sum_{i=1}^{n} \log \left(\frac{x_{i}}{\beta}\right)-\lambda \sum_{i=1}^{n}\left(\frac{x_{i}}{\beta}\right)^{c} \log \left(\frac{x_{i}}{\beta}\right) \\
& +\lambda(\alpha-1) \sum_{i=1}^{n} \frac{\left(\frac{x_{i}}{\beta}\right)^{c} e^{-\lambda\left(\frac{x_{i}}{\beta}\right)^{c}} \log \left(\frac{x_{i}}{\beta}\right)}{1-e^{-\lambda\left(\frac{x_{i}}{\beta}\right)^{c}}} \\
& \frac{\partial \ell}{\partial \beta}=-\frac{n}{\beta}-\frac{n(c-1)}{\beta}+\frac{\lambda c}{\beta} \sum_{i=1}^{n}\left(\frac{x_{i}}{\beta}\right)^{c} \\
& -\frac{(\alpha-1) \lambda c}{\beta} \sum_{i=1}^{n} \frac{\left(\frac{x_{i}}{\beta}\right)^{c} e^{-\lambda\left(\frac{x_{i}}{\beta}\right)^{c}}}{1-e^{-\lambda\left(\frac{x_{i}}{\beta}\right)^{c}}}
\end{aligned}
$$

$$
\begin{aligned}
& \frac{\partial \ell}{\partial \lambda}=\frac{n}{\lambda}-\sum_{i=1}^{n}\left(\frac{x_{i}}{\beta}\right)^{c}+(\alpha-1) \sum_{i=1}^{n} \frac{\left(\frac{x_{i}}{\beta}\right)^{c} e^{-\lambda\left(\frac{x_{i}}{\beta}\right)^{c}}}{1-e^{-\lambda\left(\frac{x_{i}}{\beta}\right)^{c}}} \\
& \frac{\partial \ell}{\partial \alpha}=\frac{n}{\alpha}+\sum_{i=1}^{n} \log \left[1-e^{-\lambda\left(\frac{x_{i}}{\beta}\right)^{c}}\right]
\end{aligned}
$$

The maximum likelihood estimates MLEs of the parameters $c, \beta, \lambda, \alpha$, can be obtained by setting the Equations (20)-(23) to zero and solving them numerically by iterative methods, such as Newton-Raphson method as there is no closed form solution to theses equations.

\section{Performance of EEWD}

In this section, the proposed model is assessed from two aspects. firstly, the performance of the MLE's is checked using a simulation study. Secondly, the goodness of fit of the EEWD is evaluated comparing to other existing distributions.

\section{Simulation Study}

To evaluate the performance of the MLEs of the EEWD obtained in section (5), we provide a Monte Carlo simulation study. The simulation is done on 1000 iteration with four different sample sizes $n=100,150$, 300,500 for the following two cases:

- $\quad$ Case I: $\alpha=0.9, \beta=2, \lambda=1.2, c=0.5$

- Case II: $\alpha=1.6, \beta=1.8, \lambda=2, c=0.9$

For each sample size $\mathrm{n}$, the MLEs are evaluated using two accuracy measures: Bias and the Mean Squared Error (MSE). Table 1 presents the MLEs together with the bias and MSE for the vector of parameters $\gamma=(\alpha, \beta$, $\lambda, c)$. It is clear that the MLEs are improved for all parameters when the sample size increases as both bias and MSE decrease on average.

\section{Real Application}

For more illustration, this section compares the efficiency of the goodness-of-fit for the EEWD distribution with some selected distributions in literature. In particular, a data set is used to compare the proposed model with: Exponential Distribution (ED), Weibull Distribution (WD), Generalized Exponential Distribution (GED), Linear Exponential Distribution (LED), A New Generalized Linear Exponential Distribution (NGLED), Modified Weibull Distribution (MWD), Exponential Weibull Distribution (EWD), Transmuted Exponential Distribution (TED) and Transmuted Linear Exponential Distribution (TLED). The data set used in comparison is provided by (Aarset, 1987) 
and represents the failure times of a sample of 50 devices. For each distribution, the MLEs and the log-likelihood function together with the Akike Information Criterion (AIC) are calculated. The data set is presented in Table 2.

Table 3 shows the results for the EEWD together with some selected distribution. It is clear that the

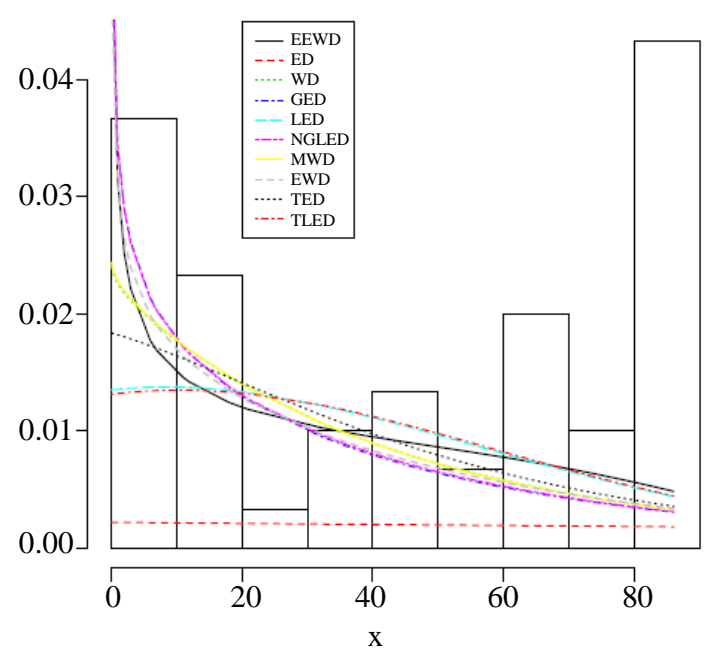

(a) proposed EEWD gives the minimum AIC and hence fits the data better than other distributions. Figure 2 shows the observed and expected frequencies and the cdf for the EEWD together with other distributions and it is plain to see that it is better at representing the data.

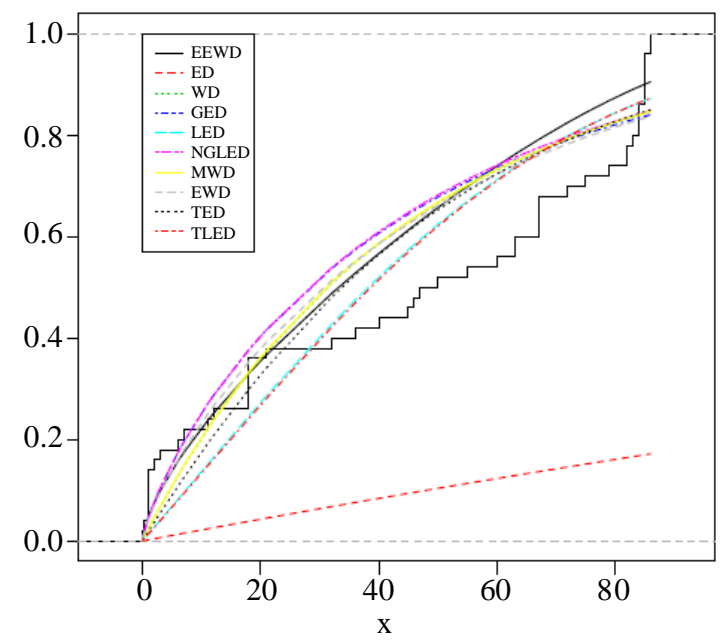

(b)

Fig. 2: Comparison of EEWD distribution with other distributions: (a) the observed and expected frequencies. (b) The cdf for each distribution

Table 1: MLEs, bias and MSE for the EEWD parameters with four different sample sizes for two different cases

\begin{tabular}{|c|c|c|c|c|c|c|c|}
\hline \multirow[b]{2}{*}{ Sample Size } & \multirow[b]{2}{*}{ Parameters } & \multicolumn{3}{|l|}{ Case I } & \multicolumn{3}{|l|}{ Case II } \\
\hline & & MLE & Bias & MSE & MLE & Bias & MSE \\
\hline \multirow[t]{4}{*}{$\mathrm{n}=100$} & $\alpha$ & 1.0198 & 0.1198 & 0.4158 & 1.8957 & 0.2957 & 1.9410 \\
\hline & $\beta$ & 2.1121 & 0.1121 & 0.5874 & 1.8000 & $3.663 \times 10^{-5}$ & 0.4740 \\
\hline & $\lambda$ & 1.2123 & 0.0123 & 0.1557 & 1.1734 & -0.0265 & 0.1809 \\
\hline & $c$ & 0.5524 & 0.0524 & 0.0413 & 0.9728 & 0.0728 & 0.0935 \\
\hline \multirow[t]{4}{*}{$\mathrm{n}=150$} & $\alpha$ & 0.9752 & 0.0752 & 0.1741 & 1.7657 & 0.1657 & 0.7769 \\
\hline & $\beta$ & 2.0987 & 0.0987 & 0.4507 & 1.8296 & 0.0296 & 0.3381 \\
\hline & $\lambda$ & 1.2289 & 0.0289 & 0.1031 & 1.1940 & -0.0059 & 0.1131 \\
\hline & $c$ & 0.5275 & 0.0275 & 0.0184 & 0.9490 & 0.0490 & 0.0553 \\
\hline \multirow[t]{4}{*}{$\mathrm{n}=300$} & $\alpha$ & 0.9234 & 0.0234 & 0.0620 & 1.6795 & 0.0795 & 0.3090 \\
\hline & $\beta$ & 2.1269 & 0.1269 & 0.2421 & 1.8515 & 0.0515 & 0.1625 \\
\hline & $\lambda$ & 1.2237 & 0.0237 & 0.0373 & 1.2182 & 0.0182 & 0.0377 \\
\hline & $c$ & 0.5160 & 0.0160 & 0.0075 & 0.9222 & 0.0222 & 0.0220 \\
\hline \multirow[t]{4}{*}{$\mathrm{n}=500$} & $\alpha$ & 0.9057 & 0.0057 & 0.0344 & 1.6672 & 0.0672 & 0.1688 \\
\hline & $\beta$ & 2.1409 & 0.1409 & 0.1649 & 1.8385 & 0.0385 & 0.0928 \\
\hline & $\lambda$ & 1.2281 & 0.0281 & 0.0241 & 1.2302 & 0.0302 & 0.0271 \\
\hline & $c$ & 0.5116 & 0.0116 & 0.0040 & 0.9083 & 0.0083 & 0.0125 \\
\hline
\end{tabular}

Table 2: Failure times of a sample of 50 devices

\begin{tabular}{|c|c|c|c|c|c|c|c|c|c|c|c|c|c|c|c|c|c|c|c|c|}
\hline Device No. & 1 & 2 & 3 & 4 & 5 & 6 & 7 & 8 & 9 & 10 & 11 & 12 & 13 & 14 & 15 & 16 & 17 & 18 & 19 & 20 \\
\hline Failure Time & 0.1 & 0.2 & 1 & 1 & 1 & 1 & 1 & 2 & 3 & 6 & 7 & 11 & 12 & 18 & 18 & 18 & 18 & 18 & 21 & 32 \\
\hline Device No. & 21.0 & 22.0 & 23 & 24 & 25 & 26 & 27 & 28 & 29 & 30 & 31 & 32 & 33 & 34 & 35 & 36 & 37 & 38 & 39 & 40 \\
\hline Failure Time & 36.0 & 40.0 & 45 & 46 & 47 & 50 & 55 & 60 & 63 & 63 & 67 & 67 & 67 & 67 & 72 & 75 & 79 & 82 & 82 & 83 \\
\hline Device No. & 41.0 & 42.0 & 43 & 44 & 45 & 46 & 47 & 48 & 49 & 50 & & & & & & & & & & \\
\hline Failure Time & 84.0 & 84.0 & 84 & 85 & 85 & 85 & 85 & 85 & 86 & 86 & & & & & & & & & & \\
\hline
\end{tabular}


Table 3: MLEs of parameters for the EEWD and SE in parentheses and some selected distributions together with the log-likelihood function and AIC for the dataset

\begin{tabular}{|c|c|c|c|}
\hline Distribution & Parameter`s Estimation & $-\ell$ & AIC \\
\hline \multirow[t]{4}{*}{$\overline{\operatorname{EEED}}(\alpha, \beta, \lambda, c)$} & $\widehat{a}=0.1525(0.0716)$ & 229.4115 & 466.832 \\
\hline & $\widehat{\beta}=67.7950(16.999)$ & & \\
\hline & $\bar{\lambda}=0.2548(0.0845)$ & & \\
\hline & $\widehat{c}=4.4488(2.027)$ & & \\
\hline $\mathrm{ED}(\beta)$ & $\widehat{\beta}=0.00219$ & 241.08 & 484.16 \\
\hline \multirow[t]{2}{*}{$\mathrm{WD}(\theta, \lambda)$} & $\hat{\theta}=0.0235$ & 241.04 & 486.08 \\
\hline & $\hat{\lambda}=0.9826$ & & \\
\hline \multirow[t]{2}{*}{$\operatorname{GED}(\alpha, \beta)$} & $\bar{\alpha}=0.7798$ & 239.99 & 483.98 \\
\hline & $\widehat{\beta}=0.0187$ & & \\
\hline \multirow[t]{2}{*}{$\operatorname{LED}(\beta, \theta)$} & $\widehat{\beta}=0.0135$ & 238.06 & 480.12 \\
\hline & $\bar{\theta}=20425 \times 10^{-4}$ & & \\
\hline \multirow[t]{4}{*}{$\operatorname{NGLED}(\alpha, \beta, \theta, \lambda)$} & $\hat{\alpha}=0.7231$ & 239.49 & 486.98 \\
\hline & $\widehat{\beta}=0.0012$ & & \\
\hline & $\hat{\theta}=0.0127$ & & \\
\hline & $\bar{\lambda}=0.1 .0682$ & & \\
\hline \multirow[t]{3}{*}{$\operatorname{MWD}(\beta, \theta, \lambda)$} & $\widehat{\beta}=0.0042$ & 241.03 & 488.06 \\
\hline & $\hat{\theta}=0.0196$ & & \\
\hline & $\hat{\lambda}=0.9759$ & & \\
\hline \multirow[t]{3}{*}{$\operatorname{EWD}(\alpha, \theta, \lambda)$} & $\widehat{\alpha}=0.6553$ & 238.71 & 483.42 \\
\hline & $\hat{\theta}=0.0076$ & & \\
\hline & $\hat{\lambda}=0.1 .1768$ & & \\
\hline \multirow[t]{2}{*}{$\operatorname{TED}(\beta, \lambda)$} & $\widehat{\beta}=0.0243$ & 240.67 & 485.34 \\
\hline & $\hat{\lambda}=-0.2436$ & & \\
\hline \multirow[t]{3}{*}{$\operatorname{TLED}(\beta, \theta, \lambda)$} & $\widehat{\beta}=0.0145$ & 238.01 & 482.02 \\
\hline & $\hat{\theta}=2.4186 \times 10^{-4}$ & & \\
\hline & $\bar{\lambda}=-0.0948$ & & \\
\hline
\end{tabular}

\section{Conclusion}

In this study, an EEWD is proposed. The distribution is constructed by compining the $\mathrm{T}-\mathrm{X}$ method along with the exponentiated method. The mathematical properties of the proposed distribution are studied. For parameters estimation, the method of ML is applied. To assess the performance of the MLEs a simulation study is conducted under various sample sizes. It is clear that the ML methods is appropriate in estimating the EEWD parameters. Moreover, real application is provided to compare the effectiveness of the EEWD compared to other known distributions in literature in terms of goodness-of-fit. The results demonstrated the efficiency of the new distribution in fitting these data accurately as it gives the minimum AIC. For future work, another estimation method can be used to estimate the model parameters; such as, Bayesian method. Moreover, other data set in different fields can be used to test the distribution's flexibility.

\section{Acknowledgement}

The author would like to thank the referee for their usefull comments.

\section{Ethics}

This article is original and contains unpublished material and no ethical issues involved.

\section{References}

Aarset, M. V. (1987). How to identify a bathtub hazard rate. IEEE Transactions on Reliability, 36(1), 106-108.

Abu-Zinadah, H. H., \& Aloufi, A. S. (2014). Some characterizations of the exponentiated Gompertz distribution. In International Mathematics Forum (Vol. 9, No. 30, pp. 1427-1439). 
Afify, A. Z., Nassar, M., Cordeiro, G. M., \& Kumar, D. (2020). The Weibull Marshall-Olkin Lindley distribution: Properties and estimation. Journal of Taibah University for Science, 14(1), 192-204.

Alzaatreh, A., Lee, C., \& Famoye, F. (2013). A new method for generating families of continuous distributions. Metron, 71(1), 63-79.

Bebbington, M., Lai, C. D., \& Zitikis, R. (2007). A flexible Weibull extension. Reliability Engineering \& System Safety, 92(6), 719-726.

Bourguignon, M., Silva, R. B., \& Cordeiro, G. M. (2014). The Weibull-G family of probability distributions. Journal of Data Science, 12(1), 53-68.

Carrasco, J. M., Ortega, E. M., \& Cordeiro, G. M. (2008). A generalized modified Weibull distribution for lifetime modeling. Computational Statistics \& Data Analysis, 53(2), 450-462.

El-Gohary, A., Alshamrani, A., \& Al-Otaibi, A. N. (2013). The generalized Gompertz distribution. Applied Mathematical Modelling, 37(1-2), 13-24.

Gupta, R. C., Gupta, P. L., \& Gupta, R. D. (1998). Modeling failure time data by Lehman alternatives. Communications in Statistics-Theory and methods, 27(4), 887-904.

Gupta, R. D., \& Kundu, D. (2001). Exponentiated exponential family: An alternative to gamma and Weibull distributions. Biometrical Journal: Journal of Mathematical Methods in Biosciences, 43(1), 117-130.

Hassan, A. S., \& Elgarhy, M. (2016). Kumaraswamy Weibull-generated family of distributions with applications. Advances and Applications in Statistics, 48(3), 205.

Klakattawi, H. S. (2019). The Weibull-gamma distribution: Properties and applications. Entropy, 21(5), 438.

Lai, C. D., Xie, M., \& Murthy, D. N. P. (2003). A modified Weibull distribution. IEEE Transactions on reliability, 52(1), 33-37.

Lee, C., Famoye, F., \& Olumolade, O. (2007). BetaWeibull distribution: Some properties and applications to censored data. Journal of modern applied statistical methods, 6(1), 17.
Mudholkar, G. S., \& Srivastava, D. K. (1993). Exponentiated Weibull family for analyzing bathtub failure-rate data. IEEE transactions on reliability, 42(2), 299-302.

Nadarajah, S., \& Kotz, S. (2006). The exponentiated type distributions. Acta Applicandae Mathematica, 92(2), 97-111.

Nadarajah, S., Cordeiro, G. M., \& Ortega, E. M. (2011). General results for the beta-modified Weibull distribution. Journal of Statistical Computation and Simulation, 81(10), 1211-1232.

Rather, A. A., \& Subramanian, C. (2018). Exponentiated Mukherjee-Islam Distribution. Journal of Statistics Applications \& Probability, 7(2), 357-361.

Sarhan, A. M., \& Apaloo, J. (2013). Exponentiated modified Weibull extension distribution. Reliability Engineering \& System Safety, 112, 137-144.

Sarhan, A. M., \& Zaindin, M. (2009). Modified weibull distribution. Applied Sciences, 11(1), 123-136.

Silva, G. O., Ortega, E. M., \& Cordeiro, G. M. (2010). The beta modified Weibull distribution. Lifetime data analysis, 16(3), 409-430.

Singla, N., Jain, K., \& Sharma, S. K. (2012). The beta generalized Weibull distribution: Properties and applications. Reliability Engineering \& System Safety, 102, 5-15.

Tahir, M. H., Cordeiro, G. M., Alizadeh, M., Mansoor, M., Zubair, M., \& Hamedani, G. G. (2015). The odd generalized exponential family of distributions with applications. Journal of Statistical Distributions and Applications, 2(1), 1.

Torabi, H., \& Montazeri, N. H. (2014). The logisticuniform distribution and its applications. Communications in Statistics-Simulation and Computation, 43(10), 2551-2569. 